\title{
Discours
}

Revue de linguistique, psycholinguistique et informatique. A journal of linguistics, psycholinguistics and computational linguistics

$1 \mid 2007$

Varia

\section{Le principe de surprise annoncée}

Grammaticalisation et pragmaticalisation de cependant

\section{Christiane Marchello-Nizia}

\section{(2) OpenEdition}

\section{Journals}

Édition électronique

URL : http://journals.openedition.org/discours/68

DOI : $10.4000 /$ discours.68

ISSN : 1963-1723

\section{Éditeur :}

Laboratoire LATTICE, Presses universitaires de Caen

\section{Référence électronique}

Christiane Marchello-Nizia, « Le principe de surprise annoncée », Discours [En ligne], 1 | 2007, mis en ligne le 11 octobre 2017, consulté le 10 décembre 2020. URL : http://journals.openedition.org/ discours/68 ; DOI : https://doi.org/10.4000/discours.68

Ce document a été généré automatiquement le 10 décembre 2020.

\section{(c) (i) (9)}

Discours est mis à disposition selon les termes de la licence Creative Commons Attribution - Pas d'Utilisation Commerciale - Pas de Modification 4.0 International. 


\title{
Le principe de surprise annoncée
}

\author{
Grammaticalisation et pragmaticalisation de cependant
}

\author{
Christiane Marchello-Nizia
}

\section{Introduction}

\subsection{Questions posées à la grammaticalisation par les marqueurs de concession}

1 Dans cette étude nous examinerons comment l'adverbe cependant s'est progressivement constitué, puis comment il a changé de sens et d'emploi, passant d'un sens temporel en ancien français au sens concessif qu'il a couramment en français moderne, et de la catégorie d'adverbe de phrase à celle de marqueur discursif supra-phrastique.

2 On n'abordera ici ni la question de l'expression de la concession en général, ni celle du sens concessif en lui-même. On centre l'analyse sur le processus de grammaticalisation qui a conduit une forme verbale (pendant) à développer, parallèlement à un emploi prépositionnel temporel, un autre emploi, adverbial celui-là, et qui de temporel à l'origine a servi dès la fin $\mathrm{du} \mathrm{xv}^{\mathrm{e}}$ siècle à exprimer la concession, tout en conservant une valeur durative. On insistera sur les étapes du processus, en mettant en évidence la nature des contextes dans lesquels se sont produites les premières occurrences (conservées) du phénomène.

3 Le phénomène examiné ici suscite plusieurs questions auxquelles on voudrait pouvoir répondre au terme, non pas d'une seule, mais de plusieurs études de ce type concernant d'autres marqueurs discursifs (MD), spécialement concessifs.

4 La première question concerne les changements sémantiques par lesquels des adverbes de SV ou de phrase acquièrent un sens pragmatique: les étapes de ce changement suivent-elles les 'chemins sémantiques' précédemment mis en évidence ${ }^{1}$ ? Ou bien les concessifs ont-ils une spécificité ? On constate en effet que certains viennent d'adverbes temporels (cependant), d'autres d'adverbes causaux (pourtant), d'autres enfin d'adverbes de manière (toutefois). Cette diversité d'origine semble assez peu fréquente. 
5 La seconde concerne le paradigme des marqueurs concessifs : tant en ancien français qu'en français actuel, ce paradigme semble formé de termes qui ont développé cette valeur secondairement, à partir d'une autre valeur première. Les MD concessifs sont-ils toujours des obtentions secondaires, comportant souvent une négation (nonbstant, malgré...) ? Ou bien partagent-ils ce caractère de 'secondarité' avec d'autres MD ?

Une dernière question concerne la définition même qu'on adopte du processus de grammaticalisation à l'origine des MD.

7 Le développement de marqueurs de discours concessifs implique à son terme la pragmaticalisation d'un terme qui auparavant n'était pas pragmatique, mais objectif, descriptif. Or de nombreuses études menées sur des faits de grammaticalisation ont montré que si la 'subjectivation' d'un terme se trouvait souvent à l'origine de tels changements, ce trait subjectif s'atténuait au fur et à mesure de la routinisation du nouveau morphème. De ce point de vue les MD sembleraient à première vue atypiques : $y$ a-t-il là contradiction? On ne reprendra pas dans les limites de cette étude le débat sur les rapports entre grammaticalisation et pragmaticalisation (Traugott, 1999, 2000), (Dostie, 2004), (Waltereit, 2006) : s'agit-il des étapes d'un même processus, ou de deux processus autonomes? Cependant, pourtant, toutefois..., concessifs ont un sens pragmatique dans la mesure où ils prennent en compte les présupposés de l'allocutaire et les inférences qu'il peut développer. Ont-ils une valeur subjective? On se contentera ici de souligner que 'pragmatique' n'est pas synonyme de 'subjectif.

\subsection{La notion de concession}

On rappelle succinctement ce qui caractérise la valeur concessive.

Selon M. A. Morel (Morel, 1996), il s'agit d'une relation A + B où B est l'inverse, le contraire de B' normalement attendu comme phénomène relié à $\mathrm{A}$. Buridant (Buridant, 2000 : chap. 26, pp. 654-672) insiste sur le fait que A (ou E1, la protase) est le premier élément d'une implication niée.

Une telle relation repose sur le fait que les interlocuteurs partagent un socle commun de convictions, suivant lequel le fait A entraîne normalement B', et non B. L'expression de la concession présuppose donc un monde de connaissances, de valeurs et de raisonnements partagés entre les locuteurs. Il s'agit bien d'un type de relation reposant sur un présupposé (comme pour puisque), tels que ceux définis par la pragmatique, et plus particulièrement d'un sous-type reposant sur un 'enchainement dialogique' (Rossari et al., 2006).

11 On a utilisé souvent pour définir la concession (Soutet, 1992:11) la notion de 'cause inefficiente, ou non efficace', qui est une forme d'implicature : ‘si q, nég. p (q étant la protase, et $\mathrm{p} \mathrm{l'apodose):} \mathrm{le} \mathrm{contraire} \mathrm{de} \mathrm{la} \mathrm{cause.} \mathrm{La} \mathrm{proposition} \mathrm{introduite} \mathrm{par} \mathrm{cependant}$ correspond à celle qui exprimerait la cause [1a], mais niée, ou inversée [1b] :

[1a] Il pleut, j'ai donc pris mon parapluie

[1b] Il pleut, cependant je n'ai pas pris mon parapluie

12 Mais en français moderne, cependant semble avoir parfois uniquement la propriété d'inversion, reliant aussi bien $\mathrm{A}$ à $\mathrm{B}$ que $\mathrm{B}$ à $\mathrm{A}$, introduisant donc soit la conséquence inattendue, soit la cause inefficiente : il marquerait alors simplement une inadéquation logique entre A et B. 
[2a] Il ne pleut pas, et cependant j'ai pris mon parapluie.

[2b] J'ai pris mon parapluie, et cependant il ne pleut pas.

\subsection{Apparition du sens concessif pour cependant}

13 En français moderne, cependant est exclusivement dédié à l'expression de la concession, comme quelques autres morphèmes (pourtant, toutefois), et contrairement à maintenant, ou enfin. Or ce n'était pas le cas dans les périodes plus anciennes.

En latin il existait des morphèmes dédiés à l'expression de la concession : etsi, etiamsi, licet, quamquam, quamvis, à côté d'autres expressions non spécifiques (cum, ou le participe présent).

En ancien français, dès l'origine neporquant, neporuec, nequedent, neportant, nonportant, sont destinés spécifiquement à l'expression de la concession, de même que seveaus et quelques autres formes. Mais malgré l'existence de ces morphèmes, entre le XIII ${ }^{\mathrm{e}}$ et le $\mathrm{XVI}^{\mathrm{e}}$ siècle quelques autres adverbes ont acquis secondairement une valeur concessive : cependant est de ceux-là, à côté de pourtant ou toutefois (Buridant, 2000). Chacun de ces nouveaux adverbes concessifs exprimait autre chose à l'origine : le temps pour cependant, le lieu puis la manière pour toutes voies > toutefois, la cause pour pourtant. Concernant cependant, l'étude menée ici vérifie bien que l'évolution est conforme à une des chaînes d'évolution sémantique repérées en grammaticalisation (cf Heine, Kuteva, 2002: 292-93: From 'temporal' to 'concessive'). A côté de ces nouveaux MD concessifs, les anciens morphèmes dédiés, neporquant, neporuec, nequedent, nonpourtant, ont disparu très vite.

\section{Cependant ${ }^{2}$}

\subsection{Grammaticalisation d'un adverbe de temps}

En ancien et moyen français, le mot cependant, comme la construction ce pendant qui en est l'origine, indiquait uniquement la durée, et plus spécifiquement la concomitance du procès énoncé dans $B$ par le verbe sur lequel portait ce morphème, avec le procès exprimé dans la phrase précédente $\mathrm{A}$; ce est ici un anaphorique faisant référence au procès énoncé dans A qui toujours implique une durée. Le syntagme ce pendant semble apparaître vers la fin $d u$ XIII $^{\mathrm{e}}$ siècle, dans un texte de nature juridique. Jusqu'au XIV ${ }^{\mathrm{e}}$ siècle, ce pendant / cependant (en un ou deux mots selon les manuscrits et les éditeurs) est introduit par la préposition en :

[3] Voirs est, - quant aucuns tient en bail et li creancier a qui les detes sont deues par la reson du bail donnent respit ou font nouveaus marchiés ou nouveles convenances de leur detes et en ce pendant l'oirs vient en aage, se li creancier vuelent l'oir poursuir, il n'en est pas en tel cas tenus a respondre. Ains convient qu'il en poursievent celui qui tint le bail...

(Philippe de Beaumanoir, Coutumes du Beauvaisis, 1283, éd. Salmon, t. I, p. 257 : 'Il est établi que, quand quelqu'un a conclu un bail et que les créanciers à qui les dettes sont dues par le fait du bail donnent des délais ou font de nouveaux marchés ou fixent de nouvelles conditions aux dettes et que pendant ce temps l'héritier devient majeur, si les créanciers veulent poursuivre l'héritier, celui-ce dans ce cas n'est pas tenu à répondre; il convient en effet que les créanciers poursuivent celui qui avait signé le bail') 
17 Comme on le verra, c'est vers le milieu du XIV ${ }^{\mathrm{e}}$ siècle que ce pendant pourra être employé absolument, comme adverbe donc. Dès le premier quart $\mathrm{du} \mathrm{xv}^{\mathrm{e}}$ siècle il va cooccurrer avec des adverbes concessifs (néanmoins cependant), et ce n'est qu'à la fin du $\mathrm{XV}^{\mathrm{e}}$ et au XVI siècle que le sens concessif apparaît clairement, se développe et finalement, au $\mathrm{XX}^{\mathrm{e}}$, supplante toute valeur temporelle.

\subsection{Origine du terme} de soi.

\subsection{Changement du sémantisme du verbe, corrélativement à l'apparition de la construction détachée : la métaphore 'espace > temps'}

figurées: 'dépendre de' chez Cicéron, 'être interrompu, suspendu' chez Virgile, 'être indécis, incertain, anxieux' (Pendet belli fortuna : Ovide, Métamorphoses, 8, 12 : 'Le sort de la guerre est incertain').

24 En latin impérial et tardif, le participe présent pendens apparaît employé comme adjectif nominalisé avec deux valeurs : concrète d'une part ('pendant'), et d'autre part abstraite ('incertain') dans le champ juridique, à savoir dans les Digesta Justiniani (VI ${ }^{\mathrm{e}} \mathrm{s}$ : : in pendenti habere aliquid 'considérer une chose comme incertaine'). 
En ancien français, pendant est très tôt utilisé comme un adjectif. Il a d'abord le sens concret et habituel du verbe : letres pendanz, seel pendant désignant un objet concret qui pend. Et le même participe pendant employé comme nom entre le $\mathrm{XII}^{\mathrm{e}}$ et le $\mathrm{xv}^{\mathrm{e}}$ siècle a également un sens spatial ('le versant d'une colline') :

[4] "Je vous dirai, fit il , comment nous eschaperons. Nous en iron, fist il, tout ce pendant aussi comme se nous devion aler vers Damas; et les Sarrazins qui la sont cuideront que nous les weillons prenre par darieres ». (Jean de Joinville, La vie de saint Louis, 1300, éd. Monfrin, p. 288 : 'Je vous dirai, fit-il, comment nous échapperons. Nous nous en irons, fit-il, tout le long de cette pente, comme si nous devions aller vers Damas, et les Sarrasins qui sont là croiront que nous voulons les prendre par derrière.')

Mais comme on l'a vu, à la fin du XIII ${ }^{\mathrm{e}}$ siècle apparaît la valeur sémantique qui nous intéresse ici: 'suspendu, non tranché, en cours'; c'est Philippe de Rémi, seigneur de Beaumanoir, qui, dans les Coutumes de Beauvaisis (1283), semble inaugurer cette nouvelle valeur de pendant. L'apparition de pendant à la fin du XIII ${ }^{\mathrm{e}}$ siècle dans le vocabulaire juridique français avec le sens figuré de 'suspendu, en suspens, en attente' qu'il avait pris justement dans le même registre lexical en latin tardif, ne serait-il pas un emprunt, lié à la prégnance du latin dans ce domaine de la culture?

\subsection{La préposition pendant : une réanalyse (réinterprétation, recatégorisation)}

Parallèlement au développement de l'adverbe cependant, on observe en ancien français celui de la forme pendant, qui, de participe présent juxtaposé à un nom et s'accordant à ce nom, se transforme par réanalyse en préposition, invariable et antéposée au nom. On voit en [5] que pendant est encore un verbe avec un complément. Syntaxiquement, si le groupe 'ce + pendant' est construit avec la préposition comme en [3], en revanche le groupe 'Nom duratif + pendant' est construit directement chez Philippe de Beaumanoir comme en [5], [6] ou [7], et chez d'autres auteurs comme en [8] :

[5] S'il avient qu'aucuns clercs ou aucune religions pledent a aucune persone par devant la justice de sainte Eglise et, de cel meisme cas, le plet pendant en la court de sainte Eglise, il vuelent pledier par devant la justice laie, la partie contre qui il pledent n'en est mie tenue a respondre devant qu'il avroient le plet de sainte Eglise delessié du tout en tout.

(Philippe de Beaumanoir, Coutumes du Beauvaisis, fin XIII ${ }^{\mathrm{e}}$ s., éd. Salmon, t. I, p. 156: 'S'il arrive qu'un religieux ou un ordre religieux fasse un procès contre quelqu'un devant la justice ecclésiastique, et que, sur ce même sujet, et alors que se déroule le procès devant le tribunal ecclésiastique, ils veuillent intenter un procès devant la justice laïque, la partie contre qui ils plaident n'est pas tenue de répondre de l'accusation avant qu'ils n'aient renoncé complètement au procès ecclésiastique')

[6]....car tout soit ainsi que li ples fust entamés au tans leur pere et li peres muert le plet pendant, avant que jugemens l'ait osté de ce dont il est saisis, li enfant demeurent en la saisine et li ples en l'estat ou il estoit quant li peres mourut.

(Philippe de Beaumanoir, Coutumes du Beauvaisis, fin XIII ${ }^{\mathrm{e}}$ s., éd. Salmon, t. I, p. 68: 'car s'il se fait que le procès ait été entamé du temps de leur père et que le père meurt durant le procès avant que le jugement l'ait libéré de 
l'accusation, les enfants restent mis en cause et le procès en l'état où il se trouvait à la mort du père')

[7] quant l'en est ajournés par devant son seigneur et, l'ajournement pendant, l'en va couchier et lever dessous autre seigneur,...

(Philippe de Beaumanoir, Coutumes du Beauvaisis, fin XIII ${ }^{\mathrm{e}}$ s., éd. Salmon, t. I, p. 110: 'Quand on est convoqué devant son seigneur, et que, à la période même de la convocation, on a sa résidence sur les terres d'un autre seigneur, ...)

$\mathrm{Au}$ cours du XIV $\mathrm{X}^{\mathrm{e}}$ siècle, pendant participe épithète d'un nom exprimant un procès duratif commence à se rencontrer, mais encore rarement, au-delà du domaine juridique :

[8] Dont il avint un jour, le siege pendant, que a l'une des portes uns tres grans assaus se fist, ...

(Jean Froissart, Chronique, 1400, éd. Diller, p. 583 : 'Il arriva un jour, pendant le siège, qu'un assaut très rude se fit contre l'une des portes [de la ville]...')

Dans toutes les constructions que nous venons d'examiner, pendant reste une forme verbale dont le sujet est le nom qui le précède, c'est une sorte d'épithète.

C'est au milieu du $\mathrm{Xv}^{\mathrm{e}}$ siècle que se produit le processus de réanalyse qui a réinterprété pendant comme une préposition. Chez un historien, Enguerrand de Monstrelet, apparaissent deux nouveautés, à côté d'emplois semblables à ceux qu'on vient de voir. D'une part pendant commence à précéder le nom construit directement: étape d'ambiguité catégorielle (épithète ou préposition ?). D'autre part, l'ex-participe devient invariable, puisqu'il apparait sous la forme pendant avec un nom féminin pluriel (on attendrait *pendanz: cf. ci-dessous). Ces deux faits montrent que le mot est désormais recatégorisé comme une préposition :

[9] Et pendant ycelles trêves, ne pourra l'une d'ycelles parties, ne ses gens, prendre [...] aulcunes villes, places, forteresses...

(Enguerrand de Monstrelet, Chronique, mi-XVe siècle, éd. p. 101 : 'Et pendant ces trêves, aucune des parties en cause, ni ses gens, ne pourront prendre aucune ville, place, forteresse..')

Mais à cette période la construction ancienne perdure et coexiste avec la nouvelle dans les mêmes textes et chez les mêmes auteurs (Monstrelet, Antoine de la Salle, etc.) :

[10] Le Roy envoiera par ycelui temps pendant devers lesdiz rois d'Espaigne et d'Escoce et ses aultres alyés...

(Enguerrand de Monstrelet, Chronique, mi-xve s., éd. p. 31 : 'Le roi enverra pendant ce temps des messagers aux rois d'Espagne et d'Ecosse et à ses autres alliés...')

2 Dès le début donc, la forme participiale pendant présentait un sens temporel duratif, étant associée à des noms marquant un processus inscrit dans la durée : plet ('procès), jugement, trêves, etc. La préposition pendant conservera cette valeur jusqu'en français moderne.

\subsection{Ce pendant : réanalyse, et du temporel-duratif ou concomitant au contraste et au concessif}

Parallèlement à cette évolution de pendant en préposition, le groupe ce pendant, qui conserve l'ancienne structure avec le verbe postposé, évolue de son côté. Dès le XIII 
siècle, pendant s'emploie avec l'anaphorique ce et la préposition en comme on l'a vu au début : en ce pendant :

[11] (reprise de [3]) Quant...li creancier a qui les detes sont deues par la reson du bail donnent respit ou font nouveaus marchiés ou nouveles convenances de leur detes et en ce pendant l' oirs vient en aage, - se li creancier vuelent l'oir poursuir, il n'en est pas en tel cas tenus a respondre.

(Philippe de Beaumanoir, Coutumes du Beauvaisis (1283), éd. Salmon, t. I, p. 257)

C'est au milieu du XIV siècle, chez Machaut (1349), que se développe cependant employé seul avec le sens temporel de 'pendant ce temps' :

[12] Li oiselès ne le doubta,

Car la prise estoit de tel guise

Faite a point et soutilment mise

$\mathrm{Qu}$ ' il y avoit un entredeus

Qui faisoit esconse entr' eaus deus,

Si qu' il ne le pouoit touchier.

Cependant $\mathrm{j}$ ' oy desclichier

La prise; a ce sceus que fu pris

Li gentils espriviers de pris.

(Guillaume de Machaut, Le Dit de l'alerion, 1349, p. 274: «L'oiselet ne redouta pas [le piège], car le piège était fait si habilement qu'il y avait un espace...Cependant j'entendis le piège se déclencher : à ce [bruit] je sus que le noble épervier était pris.')

Le subordonnant correspondant apparait chez le même auteur :

[13] Cependant qu'einsi s'ameront

[...]Leur avenroit tele aventure,

Par violence ou par nature,

Que li amans devieroit.

(Guillaume de Machaut, Le Jugement du roy de Navarre, 1349, p. 170:

'Cependant qu'ils s'aimeront ainsi..., il leur arriverait que, par un acte de

violence ou naturellement, l'amant meure..')

Ensuite le nouvel adverbe apparaît régulièrement, avec ce sens, dans Berinus (1350), chez Oresme, dans le Registre criminel du Chatelet, dans Mélusine de Jean d'Arras (1392), chez Alain Chartier, Clément de Fauquembergue (1421), chez Pierre Sala (vers 1520 : LFA), etc. Le terme est graphié soit en un seul mot, soit en deux, et parfois avec se (Pierre Sala, vers 1520 : se pendant).

Cependant indique une concomitance exacte ou partielle de deux procès. Le premier énoncé ou fragment d'énoncé $\mathrm{E} 1$ pose les bornes d'une période, à laquelle renvoie anaphoriquement ce dans la nouvelle expression, qui marque la concomitance :

[14] Et finablement fu en effect deliberé et advisé que, le plus brief que on pourroit, on rescriproit ausdis ducs de Bethford et de Bourgongne afin de prendre par leur bon conseil et advis et de leur consentement, en leur presence ou autrement, conclusion en la matiere dessusdicte; et que, cependant, on ne innoveroit riens en la façon ne en la forme desdictes lettres quousque.

(Clément de Fauquembergue, Journal, t. 2, 1421, p. 67 : '...et que, pendant ce temps, on ne changerait rien...') 
[15] ilz avoient tant fait envers luy qu' il ne feroit que demy guet, c'est assavoir depuis la mynuyt jusques au matin seulement, et que si ce pendant il vouloit venir parler a elle, elle orroit voluntiers ses devises.

(Cent nouvelles nouvelles,1460, éd. Sweetser, p. 389 : 'Ils lui avaient accordé de ne faire qu'un demi-guet, c'est à dire de minuit au matin seulement, et si pendant ce temps-là il voulait venir lui parler, elle écouterait volontiers ses propos')

Dès le premier tiers $\mathrm{du} \mathrm{Xv}^{\mathrm{e}}$ siècle, c'est surtout dans des contextes déjà concessifs que cependant devient ambigu : soit à cause de la présence d'un adverbe concessif (neantmoins, mais,..), soit dans une proposition hypothétique envisageant une situation différente comme en [16], ou résolument contraire à celle prévue [17] :

[16] La Court dit que les ouvriers et monnoiers dudit serement de l'Empire, jà receuz et qui ont fait leur espreuve en la monnoie de Paris ou en autre monnoie royal du royaume de France, pourront ouvrer et monnoier es monnoies royaulz d'icellui royaume monnoie blanche et noire, pareillement que ceulz dudit serement de France. Et, ou regard de ouvrer par lesdiz de l' Empire en monnoie $d^{\prime}$ or, les parties seront sur ce plus à plain oyes, et, ce fait, la Court leur fera droit, et se cependant est neccessité de pourveoir sur ce, les generaulz maistres des monnoies y pourverront.

(Clément de Fauquembergue, Journal, t. 2, 1421, p. 359 : Il s'agit de savoir si les ouvriers monnayeurs assermentés dans l'empire germanique ont le droit de faire de la monnaie dans le royaume de France: oui pour les monnaies 'blanches et noires', mais pour la monnaie d'or, la Cour devra réexaminer la chose; si la nécessité se présentait cependant (/pendant ce temps ?), les maîtres monnayeurs devront y pourvoir')

De même lorsque cependant se trouve auprès de l'adverbe déjà concessif néanmoins :

[17] Et tantost que ledit monseigneur le Chancellier sera retourné à Paris, on yra devers lui de par la Court lui exposer l'estat d'icelle Court pour savoir son bon plaisir et voulenté, et aussi afin qu'il ordenne du paiement des gaiges des conseilliers et officiers de ladicte Court, et que neantmoins cependant, aucuns desdis conseilliers parleront sur ce à maistre Nicaise de Bailli, clerc des tresoriers et generaulx sur le fait des finances.

(Clément de Fauquembergue, Journal, t. 3, 1431, p. 169 : En attendant que la Cour délibère au sujet des gages des conseillers de la Cour, néanmoins pendant ce temps (/ cependant ?) certains de ceux-ci devront aller parler de cette question au responsable financier.)

A la fin du $\mathrm{Xv}^{\mathrm{e}}$ siècle, il est désormais des contextes où cependant semble bien à lui seul marquer la concession: de la concomitance on peut inférer une opposition (Heine, Kuteva, $2002: 291)^{4}$, on est sur la voie de la concession: un acte qui a lieu en même temps qu'un autre mais qui va dans un sens différent et vise un but autre, a plusieurs traits communs avec une concession.

[18] Le roy leur dit: «Entretenez les...et puis nous parlerons a eulx.». Ce pendant le roy d'Angleterre, qui bien congneut que Jehan de Paris vouloit venir a la feste, commença a parler en ceste maniere.

(Le roman de Jehan de Paris,1495, éd. Wickersheimer, p. 45-46: 'Le roi leur dit: «Faites-les patienter...ensuite nous les recevrons.» Cependant, le roi d'Angleterre, qui savait bien que Jehan de Paris voulait venir à la fête, commença à parler ainsi..') 
ition de l'adverbe devient significative ; alors qu'intégré dans le SV, cependant reste temporel, en tête il devient concessif : comparez le passage ci-dessous où le sens est temporel, avec le suivant [20] ou celui ci-dessus [18] où le sens est concessif:

[19] Adoncques toutes les Dames se mirent en devoir de les arrester, et mesmes madame Salphionne cependant faict desrober les selles aux chevaulx.

(Jeanne Flore, Contes amoureux, 1537, p. 216)

[20] que une comette est apparue, et que tantost apres un prince meure, on dira qu'elle l'est venue adjourner; s'il ne s'ensuit nulle mort notable, on la laisse passer sans mot dire. Cependant je ne nie pas, lors que Dieu veut estendre sa main pour faire quelque jugement digne de memoire au monde, qu'il ne nous advertisse quelquefois par les comettes.

(Jean Calvin, Advertissement contre l'astrologie judiciaire (1549), p. 90 : Calvin se moque de ceux qui veulent à tout prix interpréter le passage des comètes de façon ad hoc; mais il reconnaît cependant que Dieu peut utiliser aussi ce moyen pour avertir les hommes.)

[21] Mais ceux qui font profession d'estre Chrestiens, et cependant nagent entre deux eaux et bigarrent la vérité de Dieu de ce que les Philosophes ont déterminé, en sorte qu'ils cherchent encores le franc-arbitre en l'homme, estant perdu et abysmé en la mort spirituelle, ceux-la, di-je, sont du tout insensez.

(Jean Calvin, Institution de la religion chrestienne,1560, I, p. 90) façon ambigüe encore, cependant peut être interprété concessivement : en cette période il devient polysémique, pouvant être encore temporel ou déjà concessif, donc à valeur pragmatique. C'est seulement à la fin de ce siècle ${ }^{5}$ semble-t-il que cependant à lui seul va porter un sens oppositif ou concessif. Calvin est le premier auteur chez qui le sens concessif de cependant domine, et souvent en cooccurrence avec mais, néantmoins, toutefois. Sur les 323 occurrences de cet adverbe dans les écrits de Calvin, il en reste cependant un petit nombre où l'adverbe a encore valeur durative: la proportion s'est nettement inversée. Quant au sens temporel il subsiste jusqu'à l'époque moderne, mais en code écrit et soutenu.

\section{Conclusion}

Les formes anciennes, neporquant/ nepourquant, neportant / nepourtant, sont encore attestées vers 1400 (Bérinus, Froissart), mais pour peu de temps.

Parallèlement au développement de cependant concessif, on voit s'intégrer dans le paradigme des MD concessifs plusieurs autres formes, à partir de lexèmes (verbe, nom ou pronom) à valeur quantifiante ou temporelle.

La voie pour certaines de ces formes, comme toutefois, sera comparable à celle de cependant: d'abord un processus de grammaticalisation qui fera passer du lexique à la grammaire une expression peu à peu routinisée, avec un sens non pragmatique. Et ensuite le développement d'une valeur pragmatique grâce à l'emploi de ces formes dans des contextes comportant par ailleurs un morphème concessif. Dès lors le morphème entre dans une phase polysémique, avant de se cantonner aux emplois concessifs. 
Un fait notable concerne surtout le français: plusieurs des morphèmes concessifs comportaient en ancien français un élément négatif (neporquant/ nepourquant, neportant / nepourtant, nonpourtant, neantmoins, ou malgré). La plupart de ces formes ont disparu, au profit de formes ne comportant pas cet élément. L'exemple le plus marquant de cette évolution est sans doute celui de pourtant, à l'origine causal, qui a 'adopté' le sens concessif de son correspondant négatif nonpourtant, encore employé à la toute fin du XVe siècle. ${ }^{6}$

\section{BIBLIOGRAPHIE}

A. BlANK, P. КоСH (eds). 1999. Historical Semantics and Cognition. Berlin: Mouton de Gruyter.

BURIDANT, C. 2000. Grammaire nouvelle de l'ancien français. Paris : SEDES.

CRAIG (GRINEVALD) C. 1991. Ways to go in Rama: a case study in polygrammaticalization. Approaches to Grammaticalization, E.C. TRAUGOTT, B. HEINE. (eds). Amsterdam: Benjamins. 455-491.

DOSTIE, G. 2004. Pragmaticalisation et marqueurs discursifs. Analyse sémantique et traitement lexicographique. Bruxelles : De Boeck.

K. FISCHER (eds). 2006. Approaches to discourse particles. Amsterdam: Elzevier.

HEINE, B. 2002. On the role of context in grammaticalization. New Reflections on Grammaticalization, I. Wischer, G. Diewald (eds). Amsterdam: Benjamins.83-101.

HEINE, B., KUTEVA, T. 2002. World Lexicon of Grammaticalization. Cambridge: Cambridge University Press.

HOPPER, P. J., TRAUGOTT, E. C. 2003/1993. Grammaticalization. Cambridge: Cambridge University Press.

MARCHELLO-NIZIA, C. 2006. Grammaticalisation et changement linguistique. Bruxelles : De Boeck. MARCHELLO-NIZIA, C. à paraître. Modéliser le changement linguistique. Romanische Forschungen. MARTIN, R., WILMET, M. 1980. Syntaxe du moyen français. Bordeaux : SOBODI.

MOREL, M-A. 1996. La concession en français. Paris : Ophrys.

RIEGEL, M., PELLAT, J-C., RIOUL R. 1994. Grammaire méthodique du français. Paris : Presses universitaires de France.

rossari, C., BeAulieu-Masson, A., Cojocariu, C., RazgouliaeVa, A. 2004. Autour des connecteurs, Réflexions sur l'énonciation et la portée. Berne : Peter Lang.

SOUTET, O. 1990. La concession en français des origines au XVI siècle. Problèmes généraux, Les tours prépositionnels. Genève : Droz.

SOUTET, O. 1992. La concession dans la phrase complexe en français des origines au XVIe siècle. Genève : Droz. 
TOBLER, A., LOMMATZSCH, E. 1925- Altfranzösische Wörterbuch. Wiesbaden. col. 634 (CD-ROM fourni par P.Blumenthal, université de Frankfurt).

TRAUGOTT, E. C. 1999. The rhetoric of counter-expectation in semantic change: a study in subjectification. Historical Semantics and Cognition, A. BLANK, Р. косн, (eds). Berlin: Mouton de Gruyter. 177-196.

TRAUGOTT, E. C. 2000. Invoking scalarity: The development of in fact. Journal of Historical Pragmatics $1 / 1: 7-25$.

TRAUGOTT, E. C., DASHER R. B. 2002. Regularity in Semantic Change. Cambridge: Cambridge University Press.

WALTEREIT, R. 2006. The rise of discourse particles in Italian: A specific type of language change. Approaches to discourse particles, K. FISCHER (eds). Amsterdam: Elzevier. 65-82.

\section{NOTES}

1. La mise au jour de telles régularités est si évidente qu'elle a conduit Heine \& Kuteva [2001) à rédiger un World Lexicon of Grammaticalization.

2. S'agissant de mener une étude sur une langue 'morte' (sans locuteur natif), le travail sur corpus est essentiel. Nous avons utilisé les bases de données suivantes: BFM (Base de Français Médiéval, ENS-LSH et UMR 'ICAR' de Lyon) : http://bfm.ens-lsh.fr/; TFA (Textes d'Ancien Français, corpus d'Ottawa) : http://www.lib.uchicago.edu/efts/ARTFL/projects/TLA/; BMF (Base de Moyen français, corpus du Dictionnaire de Moyen Français de Nancy) : http://atilf.atilf.fr/ dmf.htm ; Frantext.

3. Sur les phénomènes de polygrammaticalisation, voir C. Craig-Grinevald 1991.

4. 'Temporal > adversative'.

5. Pour Soutet, ce changement sémantique ne se produit qu'au $16^{\mathrm{e}} \mathrm{s}$.

6. Voir notre étude sur pourtant, à paraître.

\section{RÉSUMÉS}

Dans cet article, nous étudions l'émergence de l'adverbe cependant et ses divers changements de sens (de temporel en ancien français : " pendant ce temps » à concessif en français moderne) et d'emploi, ainsi que le développement de la préposition pendant. Nous constatons que l'évolution du marqueur de discours est conforme à l'une des chaînes d'évolution sémantique bien repérées dans la grammaticalisation : du temporel (concomitance) au concessif, par l'intermédiaire d'une opposition inférée. La valeur concessive commence à concurrencer la valeur temporelle au début $\mathrm{du} \mathrm{XV} \mathrm{V}^{\mathrm{e}}$ siècle avant de devenir majoritaire courant $\mathrm{XVI}^{\mathrm{e}}$ siècle.

This article examines the emergence of the adverb cependant "however" and its various meaning changes, from the temporal meaning "meanwhile" in old French to the modern concessive meaning. We also examine the grammaticalization of the preposition pendant "while", and show that this discourse marker's evolution follows a semantic chain which has already been shown to appear in various cases of grammaticalization: the evolution from time (or concomitance) to 
concession, through inferred opposition. The concessive meaning appears at the beginning of the $15^{\text {th }}$ century alongside the temporal meaning, and becomes dominant in the course of the $16^{\text {th }}$ century.

INDEX

Mots-clés : grammaticalisation, marqueurs du discours, marqueur temporel, marqueur concessif, subjectification, pragmaticalisation

Keywords : grammaticalization, discourse markers, temporal marker, concessive markers, subjectification, pragmaticalization

\section{AUTEUR}

\section{CHRISTIANE MARCHELLO-NIZIA}

ENS-LSH, UMR 5191 'ICAR’, Lyon. marchell@linguist.jussieu.fr 